\title{
Vorwort der Bearbeiter
}

Der von den Bearbeitern hier vorgelegte Text des „Thomas III“ ist das Ergebnis Jahrzehnte währender Arbeit von Benedikt Konrad Vollmann.

Die umfangreichen Materialerschließungen und Ausarbeitungen Vollmanns und seiner Mitarbeiterinnen verteilten sich zunächst auf zwei Zeiträume. Der erste umfasste die Laufzeit (1989-1995) eines DFG-Projekts im Rahmen des Sonderforschungsbereichs 226 ,Wissensvermittelnde und wissensorganisierende Literatur im Mittelalter" der Universitäten Würzburg und Eichstätt, der zweite die Jahre 1996-2011.

Im ersten Zeitraum wurde die Sammlung, Beschreibung und Analyse der Überlieferungsträger (durch Helgard Ulmschneider) weitgehend abgeschlossen. Die Kollation des Vierfüßer-Buches aus allen Handschriften, die Vollkollation von neun Handschriften und die stemmatologische Einordnung der Handschriften der Vulgata-Klasse (durch Angelika Strauß) lief dazu im Wesentlichen parallel. Im Anschluss erfolgte die Erstellung der auf dem Text von fünf Überlieferungsträgern beruhenden, schon ab 1991 von Vollmann in Umlauf gebrachten Leseausgabe.

In den zweiten Zeitraum (1995-2011) fallen sieben Publikationen Vollmanns zu mittelalterlicher Naturlehre, zu Enzyklopädik und speziell zu Überlieferung, Struktur und literarischer Technik des „Thomas III“. Die wichtigste (und zeitaufwändigste) Arbeitsleistung war jedoch die Erstellung eines die Leseausgabe ersetzenden kritischen Textes für die Bücher 1-19, der auf der Kollation von 23 Handschriften beruht. Die penibel ausgearbeitete TUSTEP-Datei hierzu enthielt den Text nebst Abkürzungsverzeichnis und Siglenliste der herangezogenen Handschriften. Die Auswahl der für die Textkonstitution berücksichtigten Handschriften erfolgte dabei auf der Grundlage der von Angelika Strauß durchgeführten Vergleichung des Quadrupedes-Buches in den 60 Vulgata-Handschriften und der von Vollmann durchgeführten Vergleichung der 12 Nicht-Vulgata-Handschriften. Da die Nicht-Vulgata-Handschriften den Quellen Thomas I-II und Folsham besonders nahe stehen, wurden sie alle als Textzeugen herangezogen. Die 60 Vertreter der Vulgata-Klasse hingegen, in der Überlieferung gekennzeichnet durch den Werkanfang 'De septem regionibus', weisen eine solche Fülle von Varianten auf, dass der Lesartenapparat damit hoffnungslos überfrachtet worden wäre. Die Ausgabe beschränkt sich deshalb darauf, für jede der sieben Untergruppen der Vulgata-Klasse einen oder mehrere Vertreter heranzuziehen. Die 12 Handschriften M1, C1, Me1, Me3, Gr1, M2, M11, M4, M7, L2, K1, L4 und im Quadrupedes-Buch zusätzlich Gr2 und M5 (Auflösung der Siglen unten S. XXIf.) sind alle in vollem Umfang bei der Textkonstitution berücksichtigt, so dass die Einbindung der Vulgata-Klasse in die Thomas III-Überlieferung hinreichend gesichert sein dürfte.

Ergänzend $\mathrm{zu}$ den bereits vorliegenden umfangreichen Arbeiten kollationierte Vollmann zusätzlich weitere neun Voll- und fünf Teilhandschriften. Er wollte seine auf dieser Basis erstellte Edition jedoch nicht publizieren ohne ihre nochmalige Kontrolle anhand aller in ihr berücksichtigten Überlieferungsträger. Dieser Vergleich sollte nach Vollmanns Meinung möglichst durch ein von außen kommendes, 'fremdes' Auge vorgenommen werden, um fehleranfällige Betriebsblindheit zu vermeiden.

Die Möglichkeit dazu ergab sich, nachdem einem bei der DFG eingereichten Antrag auf eine zweieinhalbjährige Förderung im August 2012 stattgegeben wurde. In diesem Zeitraum sollte die Kontrolle durchgeführt werden. Ferner war geplant, den von Christian Hünemörder und Janine Déus ausgearbeiteten Kommentar zur 
Leseausgabe des „Thomas III“, der ebenfalls seit Auslaufen des SFB 226 in einem leider nicht ganz vollständigen Typoskript vorlag, auf den Text der neuen Ausgabe abzustimmen und zu ergänzen. Das nur spärlich überlieferte, aber zum Thomas III-Komplex gehörende Buch 'Heilbehandlungen' (bis dahin in der Forschung als Buch *20 geführt) sollte neu kollationiert und (erstmals) in die Edition aufgenommen werden. An all diesen Schritten wollte Vollmann selbst tatkräftig mitwirken; sein nach kurzer, schwerer Leidenszeit überraschender Tod am 25. Oktober 2012 machte dies indes unmöglich.

Diese geplanten Arbeiten wurden Janine Déus übertragen und von ihr unter Mitwirkung von Rudolf Kilian Weigand durchgeführt. Déus stand zunächst vor der Aufgabe, sich ohne Möglichkeit der Rückfrage beim Herausgeber in eine hochkomplexe Überlieferungssituation einarbeiten zu müssen. Dann erfolgte die entsgungsvolle Korrektur des Variantenapparates und die anhand von 18 Voll- und 5 Teilhandschriften vorgenommene Überprüfung der ausgehobenen Lesarten, wobei jede Vollhandschrift mehr als 6200, jede Teilhandschrift ca. 2400 zu kontrollierende Stellen enthielt. Schließlich wurde die Kollation von Buch *20 durchgeführt.

Der im DFG-Antrag vorgelegte Zeitplan basierte auf der Voraussetzung der engagierten Mitwirkung des Herausgebers. Nach dem Verlust des „spiritus rector“ konnte der ursprüngliche Plan verständlicher Weise in der zur Verfügung stehenden Zeit nicht vollständig realisiert werden: Die Druckvorbereitung von Hünemörders Kommentar wird erst in einer Anschlussförderung erfolgen.

Nach der oben dargelegten notwendigen Kontrolle und Ergänzung der Ausgabe war das Überlieferungskapitel auf den neuesten Stand zu bringen. Dies hat in dankenswerter Weise und mühsamer Kleinarbeit Helgard Ulmschneider bewältigt. Für die Erläuterung der stemmatischen Zusammenhänge standen Vorarbeiten Vollmanns in Form von mehreren Aufsätzen und ergänzenden Aufzeichnungen zur Verfügung, aus denen eine verständliche Zusammenstellung eruiert werden konnte. Insbesondere galt es dabei auch, die vorhandenen divergierenden Bleistiftzeichnungen der Stemmata zusammenzuführen und in ansprechende grafische Form zu bringen. Bei all diesen Schritten hat uns Frau Dr. Gisela Vollmann-Profe in hingebungsvoller Zuarbeit unterstützt, aber auch immer wieder ihren kompetenten Rat in vielen fachlichen Fragen beigetragen. Ihr gebührt unser herzlichster Dank.

Prof. Dr. Gernot Michael Müller (Eichstätt) und seine Mitarbeiterin Johanna Schenk haben mit fachlichem Rat, Hilfe bei technischen Details und durch minutiöse Korrekturdurchsichten den Abschluss des Unternehmens mit ermöglicht. Der Aufgabe einer entbehrungsreichen Durchsicht des lateinischen Gesamttextes hat sich Dr. Konrad Goehl (Jettingen) unterzogen. Ihnen zu danken, ist uns eine angenehme Pflicht.

Die Zustimmung der Herausgeber zur Aufnahme des Bandes in die Reihe WILMA erfolgte in erfreulich rascher und unbürokratischer Form; die Verlagsleiterin Frau Ursula Reichert und ihr Team haben aus den vorgelegten Dateien das fertige Buch gestaltet. Das verdient unseren Dank. Über Jahrzehnte hinweg hat die Deutsche Forschungsgemeinschaft das Unternehmen gefördert und auch den Druck durch ihren Zuschuss erst ermöglicht. Dafür danken wir sehr.

Ob Benedikt Konrad Vollmann das Ergebnis gut geheißen hätte, wissen wir nicht; aber wir hoffen sehr, dazu beitragen zu können, dass sein Name in der Fachwelt künftig eine noch höhere Wertschätzung genießt. 